Editorial

\title{
Vigilancia de la salud pública
}

\section{Public health surveillance}

\section{Marisa Rufino San José}

Salud Laboral UGT. Madrid. España.

\author{
Correspondencia \\ Marisa Rufino San José \\ Salud Laboral UGT \\ Avda. de América, 25 \\ 28002 Madrid. España. \\ Tfno: 915890952 \\ lgarcia@cec.ugt.org
}

La salud en la empresa viene a ser un indicador, importante de la vigilancia de la Salud Pública y de sus determinantes sociales, para evitar las desigualdades en salud. El desarrollo de ésta en la empresa debe tener en cuenta el contexto socio-económico, las políticas sociales y sanitarias, las normas y valores, el estatus social del trabajador, el sistema de educación, la cohesión social, los factores biológicos y psicosociales que inciden en el sistema de atención sanitaria y la distribución a la salud y el bienestar.

Los problemas de las desigualdades en salud se acentúan en estas épocas de crisis. Afloran graves problemas, el paro de larga duración, junto a la temporalidad, precariedad e inseguridad del contrato de trabajo.

La precariedad también conlleva el menor consumo cotidiano y de bienes, con la consiguiente caída del ahorro, genera todas las patologías desde las psicosociales a las cardiovasculares y el cáncer, por las condiciones de trabajo que conlleva.

Por tanto el objetivo de conseguir la equidad, es dar más, a quien más lo necesita.

Mejorar la organización y las condiciones de trabajo, neutralizando la intensificación del trabajo que provoca riesgos psicosociales, buscando condiciones de seguridad en el empleo como punto necesario para conseguir un trabajo digno para elevar los determinantes sociales de la salud, desarrollar canales democráticos de convivencia social, con actuaciones globales en la población buscando también la cooperación internacional, OIT, OMS, UE. Y revocar el concepto empresarial de absentismo y su afán de suprimir la negociación colectiva con los trabajadores.

Los principales conceptos de salud pública asociados con el análisis del impacto de la precariedad en el empleo (Joan Bonach) son:

- Inseguridad laboral (atribuida/percibida)

- Reeducación de plantillas (dwonsizing)

- Contratos temporales/atípicos/a tiempo parcial

- Trabajo informal/economía sumergida

- Subcontratación

- Externalización

- Cambio de empleo frecuente

De ahí que la vigilancia de la salud deba programarse en función de la situación real de trabajo y subordinada, por tanto, a la evaluación de los riesgos. Sin olvidar el aspecto colectivo, que concretaremos más adelante. 


\section{VIGILANCIA DE LA SALUD}

La vigilancia de la salud según la actual denominación de la ley de Prevención de riesgos Laborales (LPRL), 1995, artículo 22, se entiende y realiza por medio de los llamados reconocimientos médicos (RM) de los trabajadores.

Siguiendo terminología de la OMS; exámenes de salud o en salud, porque en la mayoría de los casos los trabajadores están asintomáticos o como tal se perciben así mismos, en la primera impresión o punto de partida del estudio o confirmación de su estado de salud. Por ello consideramos que este "acto médico", clásico, clínico, de consulta de Medicina Interna, se trata de una aproximación al análisis y estudio del puesto de trabajo y de las condiciones laborales.

Vigilar, (según el diccionario Manuel Seco, 1990) es "observar" atentamente el funcionamiento o desarrollo de algo especialmente con intención de intervenir si no son los adecuados".

La "vigilancia de la Salud Laboral" consiste en la observación de las condiciones de trabajo y de la salud de los trabajadores, mediante la recogida y el análisis de datos sobre los factores de riesgo y la salud, la cual se realiza de manera sistemática y continua, y cuyo objetivo es la planificación y evaluación de los programas de prevención de riesgos laborales.

La vigilancia de la salud, dentro del concepto de prevención de los riesgos en el trabajo, se ha considerado, en resumen, como la función y actividad más importante de los profesionales sanitarios en el campo de la Salud Laboral y en la actualidad dentro de las funciones de los Servicios de Prevención.

Visto el problema desde una panorámica de Salud Pública, desde la perspectiva de la Epidemiología, La Sociológica del Trabajo, La Ergonomía, la vigilancia de la salud debe articularse en un concepto de prevención. Así pues la vigilancia de la salud debe ser el pilar y objetivo de la prevención con la finalidad de proteger la salud y prevenir la enfermedad.

También es un buen método de educación sanitaria y formación del trabajador.

La vigilancia individual es una estrategia preventiva y de evaluación del estado de salud del trabajador.

La vigilancia colectiva de la salud consistiría en la recopilación de datos epidemiológicos de las patologías relacionadas con el trabajo en la población activa, empresa, comunidad, para planificar la prevención y evitar los daños relacionados con el trabajo.

El objetivo principal de los reconocimientos o entrevistas laborales debe ser el análisis e identificación de los factores de riesgo en el trabajo, fase previa al programa de prevención en general o programas definidos. Por ejemplo después de realizar la evaluación de los riesgos estructurados por orden de prioridades o también apoyados en datos recientes, como los últimos accidentes de trabajo o enfermedades profesionales, etc. La vigilancia se debe tratar como método indirecto para identificar y prevenir los riesgos.

Los servicios de Prevención y en su caso los Comités de Seguridad y Salud, deben evaluar la práctica de la vigilancia de la salud, en tanto que la detección precoz, a ser posible en un período preclínico, de las enfermedades relacionadas con el trabajo, son un método válido de educación para la salud del trabajador de forma personalizada, de práctica ergonómica al estudiar y analizar el puesto de trabajo, (organización del trabajo en la dinámica trabajador-trabajo), y un sistema de vigilancia efectivo de los resultados del grado de protección de la salud de los trabajadores .

La participación de los trabajadores en su examen es cada vez más importante para conseguir resultados positivos en información, aceptación y colaboración de los mismos.

El control de la salud en el trabajo, deriva del concepto de la gestión de la prevención como derecho de los trabajadores a implantar todas las técnicas preventivas de los factores de riesgo. La entrevistas con el Médico o personal sanitario, es el comienzo de un estudio de las condiciones de trabajo y la detección precoz o preclínica de las conocidas como "enfermedades relacionadas con el trabajo" (ERT), ateniéndose al concepto acuñado por la OMS de 1985.

El examen de salud o entrevista, en resumen, faculta medidas de protección colectiva (Ergonomía y estudio de la organización del trabajo, condiciones de trabajo en la manipulación de citostáticos, control sanitario de las medidas de radioprotección, etc.). Asimismo medidas de protección individual (análisis del puesto de trabajo, información personalizada, indicaciones y metodología del examen de salud etc.). 


\section{EXAMEN DE SALUD}

Debe ser un método indirecto para identificar, evaluar y controlar los riesgos, la llamada "gestión de riesgo". Especialmente ante medidas de prevención inadecuadas y como método previo a la actuación y controles periódicos.

Como evaluación dinámica de los puestos de trabajo (análisis del proceso productivo), para estudiar:

- Métodos y organización del Trabajo.

- Sustancias químicas, (muchas cancerígenas y, muchas, sin efectos nocivos conocidos).

- Nuevas tecnologías.

- Rediseño de las condiciones de trabajo (reducción y control de riesgos).

- Resaltar el problema de los trabajadores especialmente sensibles, a determinados Riesgos.

- Realizar el control del estado de salud de los trabajadores.

- La evaluación periódica.

- Implantar y planificar un más eficaz control de riesgos.

- Nivel de cumplimiento de normas y métodos de protección de riesgos.

Como resumen, la empresa es responsable de la definición, coordinación, control y cumplimiento de la prevención de los riesgos. Es necesario el desarrollo de un compromiso compartido de planificación preventiva y gestión integral e integrada de la prevención ante los riesgos laborales.

\section{VIGILANCIA DE LA SALUD DE LA EMPRESA}

Los protocolos médicos del Ministerio tienen sus limitaciones, aunque se denominan específicos y realmente lo son. Pero si consideramos su dudosa aplicación a trabajadores asintomáticos, a los riesgos psicosociales, a el análisis del proceso productivo, y que estos parámetros son realmente el planteamiento efectivo tiene de vigilancia de la salud de la empresa. El método sistema de organización del trabajo, en la dinámica del trabajador-trabajo, considerando todos los problemas de una compleja interacción de erosión del poder empresarial.

Se precisa la formación del trabajador para su participación activa en este pacto de consenso de todas las partes, empresa, trabajador y delegados de prevención con el seguimiento de la sistemática de la prevención (artículo 15 -LRPL principios de la acción preventiva).

La llamada "nueva economía" conlleva nuevas condiciones de trabajo, la reorganización del trabajo de las nuevas tecnologías, nuevos riesgos, algunos conocidos y otros desconocidos (nanotecnologías). Es imprescindible considerar el cambio de puesto de trabajo de los trabajadores especialmente sensibles (artículo 25), buscando condiciones de trabajo compatibles con su bienestar social y cultural dentro de su contexto socio-económico.

La OMS en 1985 describió las enfermedades relacionadas con el trabajo (ERT) donde la etiología laboral no es la única, por supuesto. Pero las condiciones de trabajo pueden iniciar o agravar esta morbilidad, es caballo de batalla de la UGT el que afloren todas aquellas que hasta ahora no se declaran pero si se conocen. Para ello sería necesario la coordinación entre Sanidad y Trabajo por un lado y la necesaria formación y protocolos de actuación adecuados de los médicos del Sistema Nacional de Salud, se trataría de integrar el historial clínico laboral del trabajador.

\section{ÉTICA DE LA VIGILANCIA DE LA SALUD}

La vigilancia salvo normas y situaciones concretas es y debe ser voluntaria. La sala primera del Tribunal Constitucional así lo ratifico en el 2004.

Queda el vacio del artículo 196 de la LGSS que considera "reconocimientos previos y periódicos de los trabajadores que ocupen un puesto de trabajo en que exista un riesgo de enfermedad profesional". 
La respuesta sería minimizar o eliminar el riesgo y hacerlo compatible con la salud.

La perversión de ciertas Mutuas es querer hacer obligatoria la vigilancia para hacer caja, vigilar la selección de personal y controlar el llamado por los empresarios, absentismo laboral.

Solo cabe recordar a las asociaciones empresariales que el absentismo es un indicador de las condiciones y organización del trabajo.

|||||||||||||||||||||||||||||||||||||||||||||||||||||||||||||||||||||||||||||||||||||||||||||||||||||||||||||||||||||||||||||||||||||||||||||||||||||||||||||||||||||||||||||||||||||||||||||||||||||||||||||||||||||||||||||||||||||||||||||||||||||||||||||||||||||||||||||||||| 